\title{
EVALUACIÓN ECONÓMICA DEL COSTO DE ESTADÍA HOSPITALARIA EN UNA UNIDAD DE PACIENTE CRÍTICO
}

\author{
ECONOMIC EVALUATION OF THE COST OF HOSPITAL STAYS IN A \\ CRITICAL CARE UNIT
}

\section{AVALIAÇÃO ECONÔMICA DO CUSTO DE INTERNAÇÃO EM UMA UNIDADE DE PACIENTE CRÍTICO}

\author{
Manuel Nibaldo del Campo Rivas* \\ Katherine Alarcón Tapia** \\ Macarena Escobar Coccio*** \\ Yoana Bañados LeIro**** \\ Angélica Pilar Silva Ríos*****
}

\begin{abstract}
RESUMEN
Introducción: La Unidad de Paciente Crítico (UPC) representa un servicio de elevada complejidad. La conformación de equipos avanzados que puedan abordar la población crítica, así como la gestión del cuidado, exigen el uso eficiente de recursos humanos y financieros. Objetivo: Realizar una evaluación económica de costos asociados a la estadía hospitalaria de usuarios internados en la UPC adultos, de un hospital público de la red de salud de Chile. Material y Método: El universo estuvo conformado por 194 usuarios. Se realizó una evaluación económica que contempló costos directos, indirectos y recursos humanos, utilizando la perspectiva del prestador para el período enero-marzo 2017. Los costos totales fueron valorizados en pesos chilenos (CLP). El valor día/cama se estimó a través del cálculo proporcional del gasto total sobre el número de unidades disponibles. Resultados: El costo de estadía hospitalaria/día en UPC es de \$351.735 CLP. El servicio de procedencia que domina la usabilidad del servicio es la unidad de emergencia adultos, a la que se asocia un $47,7 \%$ del gasto mensual. El Riesgo Relativo (RR) de tener una larga estadía es mayor si el usuario proviene del servicio de emergencia adulto: $R R=1,07$ (IC 95\%: 0,51-2,26) o si cuenta con diagnóstico de sepsis $R R=2,08$ (IC 95\%: 0,91- 4,73). Conclusiones: La evaluación económica ofrece una perspectiva interesante para mejorar la gestión de los recursos a nivel hospitalario y debe ser considerada para la toma de decisiones en salud.
\end{abstract}

Palabras clave: Evaluación económica; Análisis de Costo; Cuidados intensivos; Hospitalización.

\footnotetext{
*Fonoaudiólogo, Magíster en Salud Pública. Escuela de Fonoaudiología, Facultad de Salud, Universidad Santo Tomás, Viña del Mar, Chile. ORCID: https://orcid.org/0000-0001-6076-2734. Email: mdelcampo2@santotomas.cl. Autor de correspondencia.

**Lic. Fonoaudiología. Viña del Mar, Chile. ORCID: https://orcid.org/0000-0002-0790-2678. Email: alarcontapia.k@gmail.com

***Fonoaudióloga. Viña del Mar, Chile. ORCID: https://orcid.org/0000-0001-5400-0450. Email: macarena.escobar.coccio@ gmail.com

****Enfermera. Unidad de Paciente Crítico, Hospital San Martín de Quillota, Quillota, Chile. ORCID: https://orcid.org/00000002-4135-7239. Email: yovyba@gmail.com

*****Fonoaudióloga, Magíster en Psicología. Escuela de Fonoaudiología, Facultad de Salud, Universidad Santo Tomás, Viña del Mar, Chile. ORCID: http://orcid.org/0000-0002-2116-1087. Email: asilvar@santotomas.cl
} 


\begin{abstract}
Introduction: The Critical Care Unit (CCU) represents a highly complex service. Organizing an advanced staff that can cater to critically ill patients, as well as providing care management, requires the efficient use of human and financial resources. Objective: To carry out an economic evaluation of costs associated with the hospital stay of adult users admitted to the CCU of a public hospital of the Chilean health network. Material and method: The population consisted of 194 users. An economic cost evaluation that included direct and indirect costs and human resources was carried out, using the perspective of the health provider for the period January-March 2017. The costs were calculated in Chilean pesos (CLP). The bed-day value was estimated through the proportional calculation of the total cost over the number of available units. Results: The cost of hospital stay/day in the CCU is $\$ 351,735$ CLP. The unit with the highest level of CCU usability is the adult emergency unit, which registers $47.7 \%$ of the monthly expenditures. The Relative Risk (RR) of having a lengthy stay is greater if the user comes from the adult emergency service: $R R=1.07$ (CI 95\%: 0.51-2.26) or if they have a sepsis diagnosis $\mathrm{RR}=2.08$ (CI 95\%: 0.91-4.73). Conclusions: The economic evaluation offers an interesting perspective for improving resource management at the hospital level and should be considered for health decision-making.
\end{abstract}

Key words: Economic Evaluation; Cost Analysis; Intensive Care; Hospitalization.

\title{
RESUMO
}

Introdução: A unidade de Paciente Crítico (UPC) representa um serviço altamente complexo. A formação de equipes avançadas que possam atender a população crítica, assim como a gestão do cuidado, requer o uso eficiente de recursos humanos e financeiros. Objetivo: realizar uma avaliação econômica dos custos associados à internação hospitalar de usuários internados na UPC adulto, de um hospital público da rede de saúde chilena. Material é Método: $\mathrm{O}$ universo esteve composto por 194 usuários. Foi realizada uma avaliação econômica que considerou custos diretos, indiretos e recursos humanos, da perspectiva do provedor para o período de janeiro a março de 2017. Os custos totais foram valorizados em pesos chilenos (CLP). O valor do dia/leito foi estimado através do cálculo proporcional da despesa total sobre o número de unidades disponíveis. Resultados: $\mathrm{O}$ custo de internaçáo hospitalar/dia em UPC é de \$351.735 CLP. O serviço de origem que domina a usabilidade do serviço é o pronto-socorro adulto, ao qual está associado 47,7\% da despesa mensal. O Risco Relativo (RR) de ter uma longa internação é maior se o usuário provém do serviço do pronto-socorro adulto: RR=1,07 (IC 95\%: 0,51-2,26) ou se conta com diagnóstico de sepse RR=2,08 (IC 95\%: 0,91-4,73). Conclusôes: A avaliaçáo econômica oferece uma perspectiva interessante para melhorar a gestão dos recursos no nível hospitalar e deve ser considerada para a tomada de decisóes em saúde.

Palavras-chave: Avaliação econômica; Análise de Custo; Cuidados intensivos; Hospitalização.

Fecha de recepción: 29/01/2020

Fecha de aceptación: 18/08/2020

\section{INTRODUCCIÓN}

El elevado costo de la hospitalización y el manejo del paciente crítico ${ }^{(1)}$ implican desafíos que la salud pública, por una parte, y la gestión hospitalaria, por otra $^{(2)}$, deben enfrentar. El tiempo promedio de hospitalización de un usuario en una Unidad de Paciente Crítico (UPC) varía entre 5 y 8 días $^{(3,4)}$.
En caso de observar complicaciones este lapso de tiempo puede incrementarse y aumentar el costo total de hospitalización ${ }^{(5)}$. La experiencia internacional informa que el trabajo en equipo ${ }^{(6)}$, necesario para asegurar la recuperación de funciones vitales $^{(7)}$, acelera los procesos de alta hospitalaria ${ }^{(8,9)}$, sin embargo la incorporación de profesionales no médicos a la UPC es aún incipiente en Chile ${ }^{(10)}$. 
La dotación médica ${ }^{(6)}$ y de enfermería ${ }^{(11)}$ cumplen un rol central en el manejo del usuario con riesgo vital y el apoyo de otras profesiones de la salud puede contribuir al proceso de alta de los usuarios y complejiza la provisión del servicio ${ }^{(6,12)}$.

El hecho de que un paciente requiera de más días de hospitalización implica un mayor gasto por parte de los centros hospitalarios ${ }^{(13)}$ y se vuelve relevante valorar los costos asociados a la mantención de un paciente hospitalizado en una UPC, examinando de forma paralela el flujo intrahospitalario, en lo concerniente a servicio de procedencia y tipo de diagnóstico.

Las técnicas de evaluación económica ${ }^{(14)}$ permiten medir la eficiencia relativa de las opciones implementadas en un servicio, esperando que con los mismos recursos se obtengan mejores resultados en salud ${ }^{(15)}$. Dentro de los métodos de evaluación económica se encuentra el análisis de $\operatorname{costos}^{(16)}$, minimización de costos, la evaluación de costo efectividad y la evaluación de costo utilidad, entre otras ${ }^{(17,18)}$. Debido a las diversas demandas de salud de las poblaciones y a los altos gastos incurridos en la cobertura de dichas necesidades ${ }^{(19-21)}$, es necesario evaluar económicamente procedimientos de salud y procesos hospitalarios, con el fin de aportar con evidencia para la optimización en la administración de los recursos. Según lo expuesto, el objetivo de este estudio fue analizar económicamente la estadía hospitalaria de usuarios en la UPC de un hospital de alta complejidad, de la red pública de salud.

\section{MATERIAL Y MÉTODO}

Se realizó un análisis de costos asociados a la estadía hospitalaria de usuarios internados en una UPCadulto. La perspectiva de evaluación utilizada fue la del prestador hospitalario, con una frontera temporal que consideró los meses de enero a marzo del ańo 2017. Los costos totales fueron valorizados en pesos chilenos para el mismo periodo.

El análisis económico consideró los siguientes aspectos: a) Costos directos: insumos clínicos, insumos de farmacia y exámenes; b) Costos indirectos: variables tales como luz, agua, teléfono, lavandería, otras y complementarios tales como mantención de equipamiento, edificación y compra de servicios y depreciaciones (edificio, artículos varios y equipamiento); c) Recursos humanos: médicos, enfermeras, técnicos en enfermería de nivel superior y otras remuneraciones. El valor día/ cama se estimó a través del cálculo proporcional del gasto total sobre el número de unidades disponibles.

Para complementar esta información, se analizaron los antecedentes clínicos de los usuarios atendidos, bajo la perspectiva de un estudio cuantitativo de cohorte retrospectivo ${ }^{(22)}$. Como variables de interés se contempló la edad, género, servicio de procedencia, diagnóstico del Registro Estadístico Mensual (REM) y días de hospitalización, no considerándose índice de gravedad, ni antecedentes médicos complementarios.

El universo estuvo conformado por el flujo de usuarios de la UPC adulto durante los meses de enero a marzo del año 2017 que fue de 194 usuarios. Como criterios de inclusión se estableció el disponer de la siguiente información asociada al proceso de atención: servicio de procedencia, mes de atención, género, edad y diagnóstico REM. El criterio de exclusión fue información registral incompleta. Finalmente 181 (93\%) individuos cumplieron los criterios y con ellos se conformó la muestra de estudio.

La base de datos fue validada a través de la triangulación de los antecedentes aportados por la jefatura de enfermería, revisión de fichas clínicas y departamento de finanzas del hospital, excluyéndose cualquier registro incompleto.

Para el análisis de datos se utilizó estadística descriptiva: en el caso de las variables cualitativas se aplicó análisis de frecuencia y para las cuantitativas se utilizaron medidas de tendencia central y dispersión. La distribución de las variables se analizó mediante Skewness-kurtosis Test, considerando un nivel de significancia $\mathrm{p}<0,05$ y se realizó cálculo de riesgos relativos. Para las variables que no mostraron distribución normal se utilizó la moda como medida de resumen. Las variables: Diagnóstico, servicio de procedencia y estadía, fueron estratificadas con objeto de disminuir el riesgo de sesgo. La información fue procesada inicialmente a través de Microsoft Excel y posteriormente analizada en el Stata Statistical Software 14.

Esta investigación fue aprobada por el Comité de Ética de la Universidad Santo Tomás (Código 43.18') y se pudo realizar por la asociación colaborativa con la Dirección del hospital señalado. 


\section{RESULTADOS}

La muestra estuvo compuesta por sujetos con edades entre los 15 a 94 ańos, siguiendo una distribución asimétrica negativa (-0,53 skewness y 2,4 kurtosis), con una moda de 47 años (DS: 20,1). Del total de los usuarios de la muestra el 55,8\% correspondió a personas de género masculino.

Para los meses de enero, febrero y marzo del año 2017 en el servicio de UPC, el análisis registra un costo total promedio de $\$ 147.728 .877$ pesos chilenos (Tabla 1). Utilizando los antecedentes obtenidos, se procedió a cuantificar el costo/cama mensual, considerando una disponibilidad de 14 camas en la UPC que posteriormente se evaluó el gasto diario de éstas, con un costo promedio de $\$ 351.735,42$ (Tabla 2).
La mayor parte de la ocupación de camas en la UPC se asoció a usuarios provenientes de la Unidad de Emergencia Adultos (UEA) del mismo hospital, presentando un porcentaje de utilización del presupuesto de un 47,7\%, equivalente a $\$ 70.523 .835$, seguido del servicio de Cirugía con $\$ 43.799 .013$ promedio. En tanto, los usuarios provenientes de 3 hospitales externos y maternidad, en consideración de su ocurrencia, demandaron proporcionalmente menos recursos \$2.227.068 cada uno, respectivamente (Tabla 3). El costo de estadía proporcional a la hospitalización según tipo de diagnóstico, se concentró en patologías cardiovasculares $(26 \%)$ con $\$ 38.483 .333$, y las patologías traumáticas las de menor costo total $(3,7 \%)$ asociadas a un gasto de $\$ 5.497 .619$ (Tabla 4).

Tabla 1. Costos mensuales de la estadía hospitalaria en UPC ( $n=181)$.

\begin{tabular}{lcccc}
\hline Costos $^{*}$ & Enero & Febrero & Marzo & Promedio \\
\hline Directos & 64.324 .607 & 45.089 .138 & 51.457 .829 & 53.623 .858 \\
Indirectos & 20.322 .996 & 24.292 .314 & 26.012 .877 & 23.542 .729 \\
Recursos Humanos & 74.787 .724 & 69.509 .246 & 67.389 .900 & 70.562 .290 \\
Total & 159.435 .327 & 138.890 .698 & 144.860 .606 & 147.728 .877 \\
\hline
\end{tabular}

* En pesos chilenos (CLP)

Tabla 2. Detalle costo cama mes/día ( $\mathrm{n}=181)$.

\begin{tabular}{lrrrr}
\hline Costos & Enero & \multicolumn{1}{c}{ Febrero } & \multicolumn{1}{c}{ Marzo } & \multicolumn{1}{c}{ Promedio } \\
\hline Mensual & $11.388 .237,60$ & $9.920 .764,14$ & $10.347 .186,10$ & $10.552 .062,61$ \\
Diario & $379.607,92$ & $330.692,14$ & $344.906,21$ & $351.735,42$ \\
\hline
\end{tabular}

Tabla 3. Costo según Servicio/Hospital de procedencia, enero a marzo 2017 ( $\mathrm{n}=181)$.

\begin{tabular}{lrr}
\hline Procedencia & \% & Costo \\
\hline UEA & 47,7 & 70.523 .835 \\
Cirugía & 29,6 & 43.799 .013 \\
Pabellón & 9,0 & 13.362 .410 \\
Medicina & 4,0 & 5.938 .849 \\
Otro & 3,5 & 5.196 .493 \\
Hospital 2 & 1,5 & 2.227 .068 \\
Hospital 3 & 1,5 & 2.227 .068 \\
Hospital 4 & 1,5 & 2.227 .068 \\
Maternidad & 1,5 & 2.227 .068 \\
\hline
\end{tabular}


Tabla 4. Costo según tipo de diagnóstico, enero a marzo 2017 ( $\mathrm{n}=181)$.

\begin{tabular}{lcc}
\hline Diagnóstico & \% & Costo \\
\hline Cardiovascular & 26,0 & 38.483 .333 \\
Renales/Urológico & 15,9 & 23.561 .224 \\
Otro & 14,4 & 21.205 .101 \\
Sepsis & 13,3 & 19.644 .797 \\
Digestivo & 11,2 & 16.492 .857 \\
Respiratorio & 8,5 & 12.565 .986 \\
Neurológico & 6,9 & 10.209 .863 \\
Trauma & 3,7 & 5.497 .619 \\
\hline
\end{tabular}

La estadía hospitalaria en general fue breve, con un promedio de 4.97 días (DS: 6,43). Debido a que la frecuencia de flujo se concentró en un rango acotado de hospitalización, se estratificaron los datos como sigue: a) baja estadía de 1 a 4 días, b) media estadía de 5 a 8 días y c) larga estadía, 8 días y más. Un $70 \%$ de los usuarios hospitalizados en la unidad permaneció entre 1 y 4 días en el mismo, el $17 \%$ permaneció entre 5 a 8 días y en menor porcentaje un $13 \%$ se mantuvo por 8 o más días en el servicio.

Al analizar el comportamiento de la estadía hospitalaria respecto del servicio de procedencia y diagnóstico de ingreso, se observó que el Riesgo Relativo (RR) de tener una larga estadía fue mayor si el usuario provenía del servicio de UEA del mismo hospital ( $R R=1,07$ / IC 95\%: 0,51-2,26) o si contaba con diagnóstico de sepsis $(\mathrm{RR}=2,08 / \mathrm{IC}$ 95\%: 0,91-4,73).

\section{DISCUSIÓN}

El día cama en la UPC adulto del hospital tiene un costo promedio de $\$ 351.735$ valorizados al año 2017, valor similar al reportado por un estudio previo $(\$ 374.133)^{(3)}$ a pesar de la diferencia de año (2011) y del centro hospitalario. Es importante indicar que en dicha investigación se complementaron los datos de dos centros hospitalarios, siguiendo metodológicamente un análisis retrospectivo de procesos y documentos. Cabe destacar que los usuarios provenientes del servicio de UEA fueron quienes presentaron un mayor porcentaje de utilización del presupuesto, probablemente asociado a su gravedad. En lo concerniente a los diagnósticos de internación, el mayor número de ingresos se asoció a patologías cardiovasculares con un $26 \%$, lo que coincide con el perfil epidemiológico chileno, siendo aquellas las que permanecen como principal causa de muerte en chile ${ }^{(23)}$ y en el mundo ${ }^{(24)}$. Al año 2014 en Chile, las enfermedades cardiovasculares representaron un $27,53 \%$ del total de defunciones en el país ${ }^{(25)}$ y desde una perspectiva global se ha reportado que la incidencia de estas patologías pueden incrementar los costos asociados a la atención de salud ${ }^{(26,27)}$.

La estadía promedio de los usuarios en la UPC es de 5 días, dato concordante a lo revisado por otro estudio chileno ${ }^{(13)}$. Si se aumenta la estadía hospitalaria, el costo total de la mantención de un usuario se irá incrementando, debido a los múltiples costos directos, indirectos y recursos humanos que implica dicho fenómeno ${ }^{(5)}$.

La disminución de la estadía a través del fortalecimiento del equipo profesional y la ejecución de prácticas médicas, de enfermería ${ }^{(28)}$ y de otros profesionales ${ }^{(9,29)}$, podría contribuir a generar un ahorro económico, tema que debe ser investigado empíricamente en futuros trabajos, así como los mecanismos que permitan un egreso oportuno de los usuarios del servicio. Es importante considerar la existencia de una relación ganancia/ pérdida negativa dentro de los primeros tres días de hospitalización del paciente crítico, dada la necesidad de realizar procedimientos diagnósticos y/o quirúrgicos complejos ${ }^{(30)}$.

El fortalecimiento de equipos multidisciplinarios, donde se incluya a profesionales rehabilitadores, representa una posibilidad que aún cuenta con evidencia heterogénea acerca de los resultados en salud física ${ }^{(31-33)}$ y/o cognitiva ${ }^{(34-36)}$, respectivamente. 
Se vuelve imperante conducir estudios longitudinales que permitan explorar causalidad y comprender de mejor forma los factores de riesgo vinculados a una larga estadía hospitalaria, ejecutar ensayos clínicos controlados aleatorizados que ofrezcan evidencia respecto a la efectividad del/los tratamiento/s y avanzar en la evaluación económica hacia estudios de costo-efectividad y/o costoutilidad, con el fin de aportar con información relevante en el manejo del usuario crítico.

Este estudio presenta limitaciones vinculadas a la frontera temporal en que se ejecutó y a su diseño de evaluación (análisis de costos), puesto que supone una estrategia básica para aproximarse al fenómeno de la economía en salud.

Aun así, ofrece información de base que puede ser utilizada como referencia para la proyección de estudios futuros relacionados a costo efectividad o costo utilidad respecto a intervenciones o tecnologías sanitarias específicas. Los datos reportados por esta investigación no pueden generalizarse a otros centros hospitalarios, sin considerar la complejidad y/o el perfil epidemiológico de la población a la que

\section{REFERENCIAS}

1. Rodríguez-Montoya R, Sandoval-Oliva V, CabrejoParedes J, Chacón-Angulo N, Chiquinta-Ramos G, Rodríguez-Montoya M. Variables asociadas a costos en cuidados intensivos. Rev Asoc Mex Med Crítica Ter Intensiva [Internet]. 2015 Sep [citado 3 mar 2019]; 29(3): 138-44. Disponible en: http://www.scielo.org.mx/scielo.php?script=sci_ arttext\&pid=S0187-84332015000300003\&lng=es.

2. Alvear VS, Canteros GJ, Rodríguez CP. Estudio retrospectivo de costos de tratamientos intensivos por paciente y día cama. Rev Médica Chile [Internet]. 2010 May [citado 17 ene 2019]; 138(5): 558-566. Disponible en: http://www. scielo.cl/scielo.php?script=sci_arttext\&pid=S0034$98872010000500005 \& \operatorname{lng}=$ en $\& n r m=$ iso \&tlng= en

3. Alvear S, Canteros J, Jara J, Rodríguez P. Costos reales de tratamientos intensivos por paciente y día cama. Rev Médica Chile [Internet]. 2013 Feb [citado 12 feb 2019]; 141(2): 202-8. Disponible en: https://scielo. conicyt.cl/scielo.php?script=sci_arttext\&pid=S003498872013000200009\&lng=es. http://dx.doi.org/ 10.4067/S0034-98872013000200009.

4. Lara BA, Cataldo A, Castro R, Aguilera PR, Ruiz C, atienden, ello en consideración de la heterogeneidad territorial y poblacional observada en Chile.

\section{CONCLUSIONES}

El análisis del perfil de usuario ingresado al servicio y el gasto relativo asociado a su atención suponen elementos importantes para la gestión del cuidado, en miras de contribuir a un egreso exitoso a través de la mejor toma de decisiones tanto clínicas como administrativas, basadas en la evidencia. El costo de estadía hospitalaria en UPC representa un insumo elemental para proceder a la optimización de recursos y a la toma de decisiones que puedan repercutir en la gestión hospitalaria y en el bienestar de la población. La eficiencia en la administración de recursos, así como el efecto real de los procedimientos clínicos deben explorarse en futuros estudios con objeto de contribuir al aseguramiento de la calidad de la atención y a la equidad en salud.

Andresen M. Medicina de urgencia y unidades de cuidados intensivos: Una alianza necesaria en busca de la mejoría de la atención de pacientes críticos. Rev Médica Chile [Internet]. 2016 Jul [citado 9 mar 2019]; 144(7): 911-7. Disponible en: https://scielo.conicyt. cl/scielo.php?script=sci_arttext $\&$ pid $=S 0034$ 98872016000700014\&lng=es. http://dx.doi. org/10.4067/S0034-98872016000700014.

5. Reardon PM, Fernando SM, Van Katwyk S, Thavorn K, Kobewka D, Tanuseputro P, et al. Characteristics, Outcomes, and Cost Patterns of High-Cost Patients in the Intensive Care Unit. Crit Care Res Pract [Internet]. 2018 Sep [citado 27 ene 2019]; 1-7. Disponible en: https://www.ncbi.nlm. nih.gov/pmc/articles/PMC6139208/

6. Ervin JN, Kahn JM, Cohen TR, Weingart LR. Teamwork in the Intensive Care Unit. Am Psychol [Internet]. 2018 May-Jun [citado 2 ene 2019]; 73(4): 468-77. Disponible en: https://pubmed. ncbi.nlm.nih.gov/29792461/

7. Baumgartner CA, Bewyer E, Bruner D. Management of Communication and Swallowing in Intensive Care: The Role of the Speech Pathologist. AACN Adv Crit Care [Internet]. 2008 Oct-Dec [citado 3 mar 2019]; 19(4): 433-43. Disponible en: https:// pubmed.ncbi.nlm.nih.gov/29792461/ 
8. McRae J. The role of speech and language therapy in critical care. ICU Manag Pract [Internet]. 2018 Sep [citado 5 may 2019]; 18(2): 128-31. Disponible en: https://healthmanagement.org/c/ icu/issuearticle/the-role-of-speech-and-languagetherapy-in-critical-care

9. Dong Z, Yu B, Sun Y, Fang W, Li L. Effects of early rehabilitation therapy on patients with mechanical ventilation. World J Emerg Med [Internet]. 2014 Ene [citado 8 ago 2019]; 5(1): 48-52. Disponible en: https://www.ncbi.nlm.nih.gov/pmc/articles/ PMC4129870/

10. SOCHIMI. La Sociedad Chilena de Medicina Intensiva incorpora nuevas disciplinas a su organización: fonoaudiología y terapia ocupacional [Internet]. 2018 [citado 17 ene 2019]. Disponible en: https://www.medicina-intensiva.cl/

11. Eaton TL, McPeake J, Rogan J, Johnson A, Boehm LM. Caring for Survivors of Critical Illness: Current Practices and the Role of the Nurse in Intensive Care Unit Aftercare. Am J Crit Care Off Publ Am Assoc Crit-Care Nurses [Internet]. 2019 Nov [citado 27 dic 2029]; 28(6): 481-5. Disponible en: https://pubmed.ncbi.nlm.nih.gov/31676524/

12. Kendall-Gallagher D, Reeves S, Alexanian JA, Kitto S. A nursing perspective of interprofessional work in critical care: Findings from a secondary analysis. J Crit Care [Internet]. 2017 Abr [citado 12 mar 2019]; 38: 20-6. Disponible en: https://pubmed. ncbi.nlm.nih.gov/31676524/

13. Ruiz C, Díaz MÁ, Zapata JM, Bravo S, Panay S, Escobar C, et al. Características y evolución de los pacientes que ingresan a una Unidad de Cuidados Intensivos de un hospital público. Rev Médica Chile [Internet]. 2016 Oct [citado 28 ene 2020]; 144(10): 1297-304. Disponible en: https://scielo.conicyt. cl/scielo.php?script=sci_arttext \&pid=S003498872016001000009\&lng=es. http://dx.doi. org/10.4067/S0034-98872016001000009.

14. Dang A, Likhar N, Alok U. Importance of Economic Evaluation in Health Care: An Indian Perspective. Value Health Reg Issues [Internet]. 2016 May [citado 28 ene 2020]; 9: 78-83. Disponible en: https://pubmed.ncbi.nlm.nih.gov/27881265/

15. Castillo-Riquelme M, Zárate V. La evaluación económica en salud. Rev Médica Chile [Internet]. 2010 Sep [citado 28 ene 2020]; 138(Supl. 2): 6767. Disponible en: https://scielo.conicyt. cl/scielo.php?script=sci_arttext\&pid=S003498872010001000001\&lng=es. http://dx.doi. org/10.4067/S0034-98872010001000001.

16. Lenz-Alcayaga, R. Análisis de costos en evaluaciones económicas en salud: Aspectos introductorios. Rev Med Chile [Internet]. 2010 Sep [citado 28 nov 2019]; 138 (Supl. 2): 88-92. Disponible en: https://scielo.
conicyt.cl/scielo.php?script=sci_arttext\&pid=S003498872010001000006\&lng=es. http:// dx.doi . org/10.4067/S0034-98872010001000006.

17. Zárate V. Evaluaciones económicas en salud: Conceptos básicos y clasificación. Rev Med Chile [Internet]. 2010 Sep [citado 28 nov 2019]; 138(Supl. 2): 93-97. Disponible en: https://scielo.conicyt. $\mathrm{cl} /$ scielo.php?script=sci_arttext $\&$ pid $=$ S003498872010001000007\&lng=es.

18. Rudmik L, Drummond M. Health economic evaluation: important principles and methodology. Laryngoscope [Internet]. 2013 Jun [citado 12 ene 2020]; 123(6): 1341-1347. Disponible en: https:// pubmed.ncbi.nlm.nih.gov/23483522/

19. Roman-Urrestarazu A, Yang JC, Ettelt S, Thalmann I, Seguel Ravest V, Brayne C. Private health insurance in Germany and Chile: two stories of coexistence, segmentation and conflict. Int J Equity Health [Internet]. 2018 Ago [citado 28 ene 2020]; 17: 112-126. Disponible en: https://www.ncbi. nlm.nih.gov/pmc/articles/PMC6091104/

20. Mondaca ALN, Chi C. Equity in out-of-pocket payment in Chile. Rev Saúde Pública [Internet]. 2017 May [citado 28 ene 2020]; 51: 44-56. Disponible en: https://www.ncbi.nlm.nih.gov/ pmc/articles/PMC5433787/

21. Papanicolas I, Woskie LR, Jha AK. Health Care Spending in the United States and Other HighIncome Countries. JAMA [Internet]. 2018 Mar [citado 28 ago 2019]; 319(10): 1024-39. Disponible en: https://jamanetwork.com/journals/jama/ article-abstract/2674671

22. Hernández SR, Fernández CC, Baptista LP. Metodología de la investigación. 5a ed. México, D.F.: McGraw-Hill; 2010. 613 p.

23. Lanas F, Serón P, Puig T. Evolución del riesgo cardiovascular y sus factores en Temuco entre 1989 y 2011-12. Rev Médica Chile [Internet]. 2014 Abr [citado 28 ene 2020]; 142(4): 46774. Disponible en: https://scielo.conicyt. $\mathrm{cl} /$ scielo.php?script $=$ sci_arttext $\&$ pid $=$ S003498872014000400008\&lng=es. http://dx.doi. org/10.4067/S0034-98872014000400008.

24. Jokinen E. Obesity and cardiovascular disease. Minerva Pediatr [Internet]. 2015 Feb [citado 28 ene 2020]; 67(1): 25-32. Disponible en: https:// pubmed.ncbi.nlm.nih.gov/25387321/

25. MINSAL. Las enfermedades cardiovasculares [Internet]. Ministerio de Salud - Gobierno de Chile. 2017 Ago [citado 22 ene 2019]. Disponible en: https://www.minsal.cl/mes-del-corazon-2017/

26. Bansilal S, Castellano JM, Fuster V. Global burden of CVD: focus on secondary prevention of cardiovascular disease. Int J Cardiol [Internet]. 2015 Dic [citado 28 ene 2020]; 201 (Supl.1): S1- 
S7. Disponible en: https://pubmed.ncbi.nlm.nih. gov/26747389/

27. Balbay Y, Gagnon-Arpin I, Malhan S. Modeling the burden of cardiovascular disease in Turkey. Anatol J Cardiol [Internet]. 2018 Oct [citado 12 ene 2020]; 20(4): 235-240. Disponible en: https://www.ncbi. nlm.nih.gov/pmc/articles/PMC6249532/

28. Woo B, Lee J, Tam W. The impact of the advanced practice nursing role on quality of care, clinical outcomes, patient satisfaction, and cost in the emergency and critical care settings: a systematic review. Hum Resour Health [Internet]. 2017 Sep [citado 28 ene 2020]; 15(1): 63-85. Disponible en: https://www.ncbi.nlm.nih.gov/pmc/articles/ PMC5594520/

29. Silva D, Lira F, Oliveira J, Canuto M. Speech therapy practice in the intensive care unit of a hospital of infectious diseases of Alagoas. Rev CEFAC [Internet]. 2016 Feb [citado 26 dic 2019]; 18(1): 174-83. Disponible en: http://www.scielo. br/scielo.php?script=sci_arttext $\&$ pid $=$ S1516$18462016000100174 \& \operatorname{lng}=$ en.

30. Kılıç M, Yüzkat N, Soyalp C, Gülhaş N. Cost Analysis on Intensive Care Unit Costs Based on the Length of Stay. Turk J Anaesthesiol Reanim [Internet]. 2019 Ene [citado 28 ene 2020]; 47(2): 142-5. Disponible en: https://turkjanaesthesiolreanim.org/ en/cost-analysis-on-intensive-care-unit-costs-basedon-the-length-of-stay-1324

31. Reid JC, Unger J, McCaskell D, Childerhose L, Zorko DJ, Kho ME. Physical rehabilitation interventions in the intensive care unit: a scoping review of 117 studies. J Intensive Care [Internet]. 2018 Dic [citado 27 ene 2020]; 6: 80-92. Disponible en: https://www.ncbi.nlm.nih.gov/ pmc/articles/PMC6286501/

32. Taito S, Sanui M, Yasuda H, Shime N, Lefor
AK. Current rehabilitation practices in intensive care units: a preliminary survey by the Japanese Society of Education for Physicians and Trainees in Intensive Care (JSEPTIC) Clinical Trial Group. J Intensive Care [Internet]. 2016 Oct [citado 20 ene 2020]; 4: 66-9. Disponible en: https://www.ncbi. nlm.nih.gov/pmc/articles/PMC5084398/

33. Castro-Avila A, Serón P, Fan E, Gaete M, Mickan S. Effect of Early Rehabilitation during Intensive Care Unit Stay on Functional Status: Systematic Review and Meta-Analysis. PLoS ONE [Internet]. 2015 Jul [citado 12 jul 2019]; 10(7): 1-21. Disponible en: https://www.ncbi.nlm.nih.gov/pmc/articles/ PMC4488896/

34. Jackson J, Ely E, Morey M, Anderson V, Siebert C, Denne L, et al. Cognitive and Physical Rehabilitation of ICU Survivors: results of the RETURN randomized, controlled pilot investigation. Crit Care Med [Internet]. 2012 Abr [citado 14 ene 2020]; 40(4): 1088-97. Disponible en: https:// pubmed.ncbi.nlm.nih.gov/22080631/

35. Brummel N, Jackson J, Girard T, Pandharipande P, Schiro E, Work B, et al. A Combined Early Cognitive and Physical Rehabilitation Program for People Who Are Critically Ill: The Activity and Cognitive Therapy in the Intensive Care Unit (ACT-ICU) Trial. Phys Ther [Internet]. 2012 May [citado 05 ene 2020]; 92(12): 1580-92. Disponible en: https:/www.ncbi.nlm.nih.gov/pmc/articles/ PMC3513484/

36. Zhao J, Yao L, Wang C, Sun Y, Sun Z. The effects of cognitive intervention on cognitive impairments after intensive care unit admission. Neuropsychol Rehabil [Internet]. 2017 Abr [citado 16 ene 2020]; 27(3): 301-17. Disponible en: https://pubmed. ncbi.nlm.nih.gov/26313129/ 